Published in final edited form as:

J Urol. 2020 May ; 203(5): 977. doi:10.1097/JU.0000000000000656.02.

\title{
REPLY BY AUTHORS
}

\author{
Heidi S Harvie ${ }^{1}$, Cindy L Amundsen ${ }^{2}$, Simon J Neuwahl ${ }^{3}$, Amanda A Honeycutt ${ }^{3}$, Emily S \\ Lukacz $^{4}$, Vivian W Sung ${ }^{5}$, Rebecca G Rogers ${ }^{6,7}$, David Ellington ${ }^{8}$, Cecile A Ferrando ${ }^{9}$, \\ Christopher J Chermansky ${ }^{10}$, Donna Mazloomdoost ${ }^{11}$, Sonia Thomas ${ }^{3}$
}

${ }^{1}$ Department of Obstetrics and Gynecology, University of Pennsylvania, Philadelphia, Pennsylvania. ${ }^{2}$ Department of Obstetrics and Gynecology, Duke University, Durham. ${ }^{3}$ Social, Statistical and Environmental Sciences Group, RTI International, Research Triangle Park, North Carolina. ${ }^{4}$ Department of Obstetrics, Gynecology \& Reproductive Sciences, University of California San Diego, La Jolla, California. ${ }^{5}$ Department of Obstetrics and Gynecology, Brown University, Providence, Rhode Island. ${ }^{6}$ Department of Obstetrics and Gynecology, University of New Mexico, Albuquerque, New Mexico. ${ }^{7}$ Department of Women's Health, Dell Medical School, University of Texas, Austin, Texas. ${ }^{8}$ Department of Obstetrics and Gynecology, University of Alabama at Birmingham, Birmingham, Alabama. ${ }^{9}$ Obstetrics, Gynecology and Women's Health Institute, Cleveland Clinic, Cleveland, Ohio. ${ }^{10}$ Department of Urology, University of Pittsburgh, Pittsburgh, Pennsylvania. ${ }^{11}$ Eunice Kennedy Shriver National Institute of Child Health and Human Development, National Institutes of Health, Bethesda, Maryland.

Subsequent to the publication of the guideline by Gormley et al (reference 10 in article) several studies have reported continued BTX efficacy and improved quality of life while maintaining the same median inter-injection intervals beyond 2-year followup. ${ }^{1-3}$ Causes for therapy discontinuation are most accurately reported in prospective studies. Thus, as referenced in the comment, the 3.5-year followup in the study by Nitti et al showed that $5.7 \%$ of discontinuations were due to lack of efficacy and the highest rate, $12 \%$, was due to trial burden. ${ }^{4}$ For the 22 BTX participants who did not complete the study protocol a sensitivity analysis showed that assuming maximum BTX costs for all would raise 2-year BTX costs to almost $\$ 13,000$, compared to 2-year SNM costs of $\$ 35,680$ (supplementary Appendix C, https://www.jurology.com).

The ROSETTA study accommodated for advancements in lead technology and minimal stimulation thresholds during lead placement were incorporated in the study design, a novel concept for an InterStim ${ }^{\circledR}$ study. Thus, optimal lead placement was assessed by the high clinical responder rate (84\%), low revision and removal rates (3\% and 8.6\%) and 2-year sustained efficacy (reference 14 in article).

Threshold analysis evaluated the impact of single stage vs 2-stage SNM implantation and length of SNM technology life. It showed that a single stage implantation technique would reduce SNM costs by $15 \%$ compared to 2 -stage, but importantly this did not include implantation and explantation costs for the trial's nonresponders. In addition, the assumption of rechargeable SNM technology would result in similar costs for SNM and BTX by 39 years for 2 -stage and by 25 years for single stage implantation. 


\section{REFERENCES}

1. Ginsberg DA, Drake MJ, Kaufmann A et al.: Long-term treatment with onabotulinumtoxinA results in consistent, durable improvements in health related quality of life in patients with overactive bladder. J Urol 2017; 198: 897. [PubMed: 28536084]

2. Dowson C, Watkins J, Khan MS et al.: Repeated botulinum toxin type A injections for refractory overactive bladder: medium-term outcomes, safety profile, and discontinuation rates. Eur Urol 2012; 61: 834. [PubMed: 22204745]

3. Veeratterapillay R, Hardling C, Teo L et al.: Discontinuation rates and inter-injection interval for repeated intravesical botulinum toxin type A injection for detrusor overactivity. Int J Urol 2014; 21 : 175. [PubMed: 23819724]

4. Nitti VW, Ginsberg D, Sievert KD et al.: Durable efficacy and safety of long-term onabotulinumtoxinA treatment in patients with overactive bladder syndrome: final results of a 3.5year study. J Urol 2016; 196: 791. [PubMed: 27038769] 